\title{
Intususcepción como causa de obstrucción intestinal en el adulto: un desafío para los cirujanos
}

\author{
Intussusception as cause of intestinal obstruction in the adult: \\ a challenge for surgeons \\ Fernando Aguirre1 , Álvaro Silva¹, Patricia Parra, Diego Salcedo², Paula López², \\ Yulieth Acevedo $^{3}$ \\ 1 Médico, cirujano general, Hospital Simón Bolívar-Universidad El Bosque, Bogotá, D.C., Colombia \\ 2 Médico, residente de Cirugía General, Hospital Simón Bolívar-Universidad El Bosque, Bogotá, D.C., Colombia \\ 3 Médica interna de Cirugía General, Hospital Simón Bolívar-Universidad Antonio Nariño, Bogotá, D.C., Colombia
}

\section{Resumen}

La intususcepción en adultos es una condición inusual, que corresponde al $5 \%$ de todos los casos de obstrucción intestinal y carece de síntomas específicos. El diagnóstico preoperatorio sigue siendo un desafío para el cirujano y el tratamiento óptimo de esta condición es controvertido.

Se presentan dos casos clínicos de intususcepción con diferente etiología. El primero sufrió una intususcepción colocólica por un adenocarcinoma y, el segundo, una intususcepción ileocólica por un tumor del estroma gastrointestinal (GIST). Los casos fueron diagnosticados preoperatoriamente mediante tomografía axial de abdomen. En ambos casos, se practicó una resección quirúrgica oncológica. El estudio de histopatología confirmó el diagnóstico definitivo.

Se describen el diagnóstico y el tratamiento de la intususcepción como causa de obstrucción intestinal en los adultos, y se presenta una revisión de la literatura científica.

Palabras clave: intususcepción; obstrucción intestinal; adenocarcinoma; tumores del estroma gastrointestinal; diagnóstico diferencial; tomografía computarizada.

\footnotetext{
Abstract

Intestinal intussusception in adults is a rare finding, with non specific symptoms and is responsible for $5 \%$ of intestinal obstructions. Pre-operative diagnosis remains a challenge for surgeons and the optimal treatment is still in debate.

We present two cases of intestinal intussusception caused by different etiologies; the first is a colon-colonic intussusception caused by an adenocarcinoma, and the second is an ileo-colonic intussusception caused by a gastrointestinal stromal tumor (GIST). Both cases were diagnostic pre-operatively by abdominal CT. Oncologic surgical

Fecha de recibido: 10/05/2018. Fecha aceptación: 28/05/2018.

Correspondencia: Diego Salcedo, Avenida carrera 9 N¹31A-02 Teléfono: (571) (320) 800-7318, Bogotá, D.C., Colombia

Correo electrónico: dsalcedo_ub@hotmail.com

Citar como: Aguirre F, Silva Á, Parra P, Salcedo D, López P, Acevedo Y. Intususcepción como causa de obstrucción intestinal en el adulto: un desafío para los cirujanos. Rev Colomb Cir. 2019;34:79-86. https://doi.org/10.30944/20117582.102

Este es un artículo de acceso abierto bajo una Licencia Creative Commons - BY-NC-ND https://creativecommons.org/licenses/by-nc-nd/4.0/deed.es
} 
resection was performed in both cases. Histopathology report confirmed neoplastic tissues. We describe the diagnosis and treatment of intestinal intussusception as a cause of intestinal obstruction in the adult population.

Key words: intussusception; intestinal obstruction; adenocarcinoma; gastrointestinal stromal tumors; diagnosis, differential; computed tomography.

\section{Introducción}

La intususcepción, o invaginación intestinal, es la introducción de una parte del intestino dentro de la luz de un segmento adyacente, lo cual causa obstrucción intestinal mecánica. Según su ubicación anatómica, se clasifica en cuatro tipos: cólica, cuando compromete el colon; entérica, cuando compromete solo el intestino delgado; ileocecal, en la cual la válvula ileocecal actúa como punto guía de la intususcepción, e ileocólica, en la cual elíleon terminal se invagina a través de la válvula ileocecal hacia el colon ${ }^{\mathrm{I}}$. Cualquier aumento del volumen de los tejidos en la luz intestinal, ya sea por inflamación de la mucosa o por una lesión con efecto de masa, puede actuar como punto de partida; esto, acompañado de una actividad hiperperistáltica, ocasiona que un segmento de intestino, posiblemente junto con su mesenterio, se proyecte hacia la luz intestinal adyacente ${ }^{2}$.

La intususcepción es una causa infrecuente de obstrucción intestinal en el adulto, en comparación con el grupo pediátrico ${ }^{3}$. La literatura informa una incidencia anual en adultos tan baja como 2 casos por Ioo.oooo personas. Solo el $5 \%$ de las intususcepciones confirmadas se presentan en adultos y, en ellos, el $90 \%$ se debe a un proceso patológico. De estos, el $60 \%$ son neoplasias, en contraste con la población pediátrica en la que la etiología suele ser benigna o fisiológica ${ }^{4}$. Los pacientes pediátricos suelen presentar la tríada clínica clásica de heces en jarabe de grosella, dolor abdominal de tipo cólico y masa abdominal palpable; sin embargo, los adultos, aunque suelen presentar manifestaciones clínicas de obstrucción intestinal, carecen de síntomas específicos, lo que representa un desafío para los cirujanos ${ }^{5}$.

El uso generalizado de la tomografía computadorizada (TC) de abdomen ha llevado a una mayor detección de intususcepción en la población en general ${ }^{6}$. Esta técnica alcanza una sensibilidad del $58 \%$ al Ioo \% y una especificidad del $57 \%$ al $7 \mathrm{I} \%$ en el reconocimiento de la intususcepción intestinal 7 ; no obstante, en la literatura se informa que solo el o,04 \% de todos los casos de intususcepción detectados por TC abdominal se presenta en adultos ${ }^{8}$.

Como la intususcepción es infrecuente en la población adulta, pero una entidad quirúrgicamente importante, a continuación, se presentan dos casos de la experiencia de nuestro hospital y una revisión de la literatura científica.

\section{Reporte de casos}

Caso I. Se trata de un hombre de 64 años de edad que ingresó al servicio de urgencias con un cuadro clínico de seis meses de evolución de dolor abdominal intermitente, náuseas y vómitos. De interés, solo existía el antecedente de una laparotomía exploratoria por herida penetrante de abdomen por arma de fuego, sin perforación intestinal.

En el examen físico, el abdomen estaba ligeramente distendido, sin signos de irritación peritoneal. En el mesogastrio, se palpaba una masa de $4 \times 7 \mathrm{~cm}$ de diámetro. Los exámenes de laboratorio no presentaron anormalidades. En la radiografía abdominal se observaron signos de obstrucción intestinal con asas dilatadas de intestino delgado. En la TC abdominal se encontró un patrón obstructivo con niveles hidroaéreos e importante dilatación de las asas del intestino delgado, que se relacionó con una intususcepción del colon transverso en el colon descendente (colocólica) (figura I).

En la laparotomía exploratoria se encontró una intususcepción del colon derecho, que atravesaba el colon transverso y alcanzaba parte del 

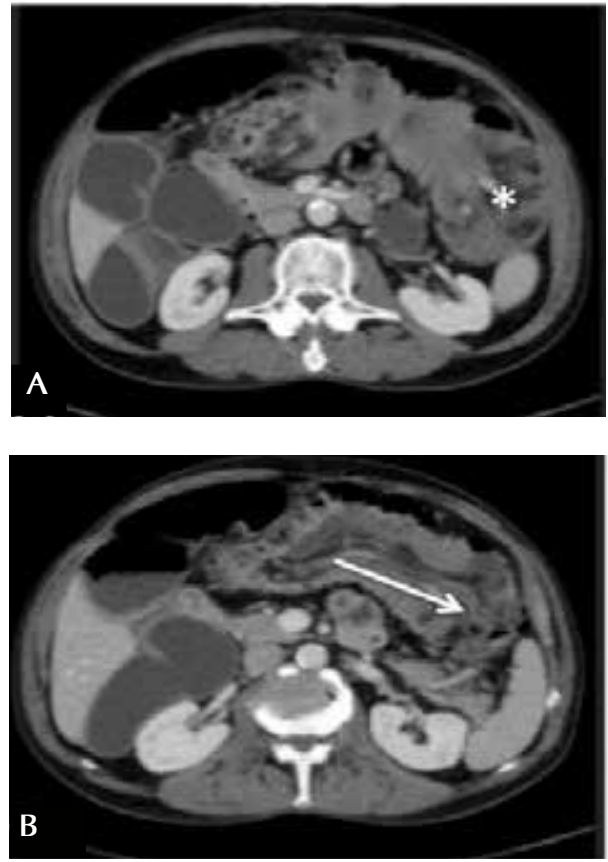

Figura 1. TC abdominal, corte axial. A. Masa sobre el colon transverso (asterisco). B. Origen de la intususcepción (flecha) en el colon transverso y extensión hacia el colon descendente

colon descendente (figura 2). Tras la reducción manual, se palpó una masa bien delimitada, de $5 \mathrm{~cm}$ de diámetro, en el colon transverso. Se practicaron una hemicolectomía derecha con principios oncológicos y una anastomosis ileocólica. El periodo posoperatorio transcurrió sin complicaciones y el paciente fue dado de alta a los cinco días de la intervención. El examen histopatológico del colon resecado reveló una masa de $2 \times 5 \mathrm{~cm}$ correspondiente a un adenocarcinoma mucinoso (figura 3 ).

Caso 2. Se trata de un hombre de 55 años de edad que ingresó al servicio de urgencias con un cuadro clínico de dos años de evolución de dolor abdominal intermitente que se exacerbó en las últimas semanas, sin pérdida de peso. No tenía antecedentes quirúrgicos abdominales, ni otros antecedentes personales ni familiares de interés.

En el examen físico, el abdomen estaba distendido, doloroso a la palpación derecha, sin signos de irritación peritoneal. En el hemiabdomen derecho, se palpaba una masa de $2 \times 5 \mathrm{~cm}$

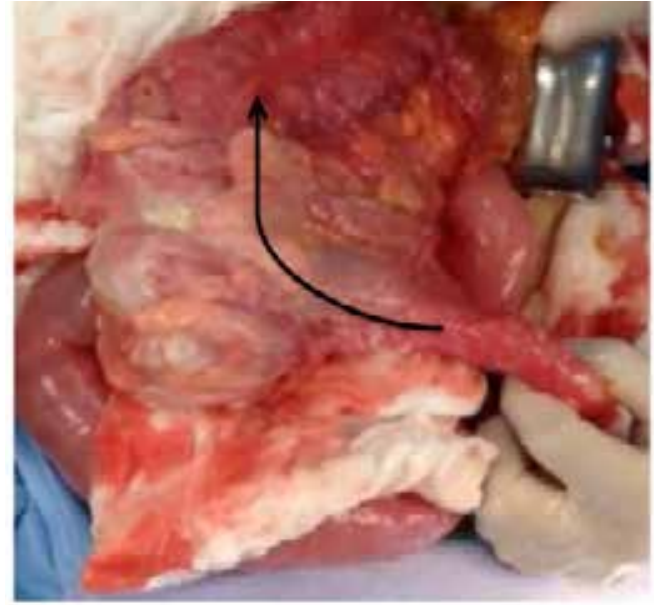

Figura 2. Pieza quirúrgica: intususcepción colocólica en el colon transverso derecho hacia el colon descendente (flecha).
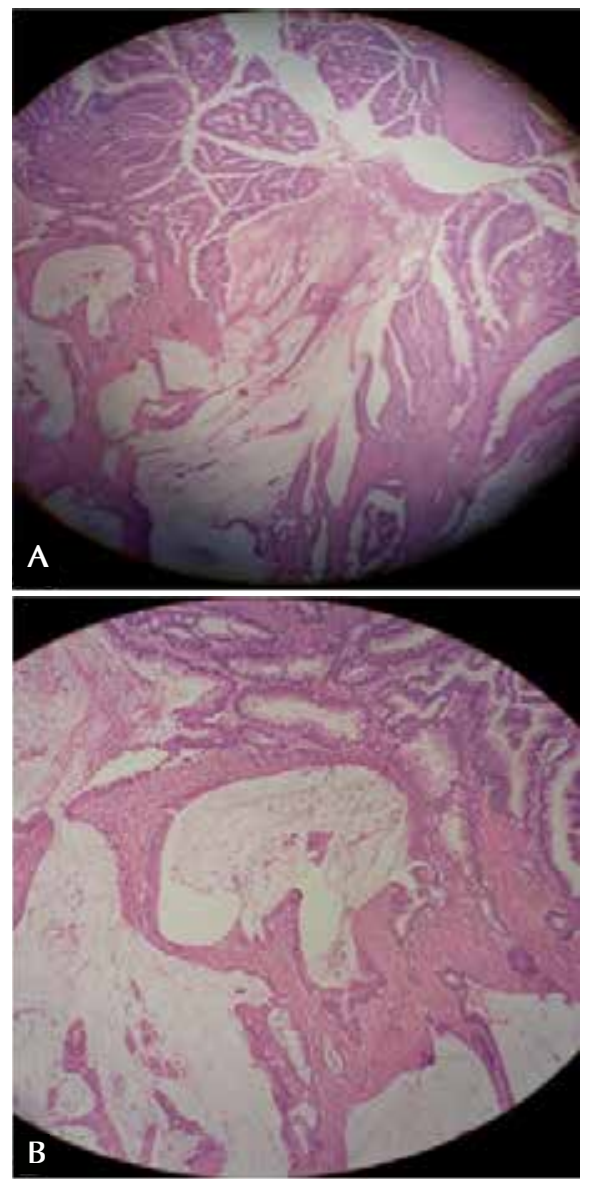

Figura 3. A y B. Adenocarcinoma moderadamente diferenciado con componente mucinoso. Hematoxilina y eosina, 200X. 
de diámetro. En los exámenes de laboratorio no se encontraron anormalidades. En la radiografía abdominal no se observó dilatación de asas. En la TC abdominal se apreció una masa de contornos lobulados en la región ileocólica, con intususcepción de 4,8 x $5 \mathrm{~cm}$, con el 'signo de la diana' presente (figura 4 ).

Por persistencia del dolor abdominal, se decidió practicar una laparotomía exploratoria, en la que se encontró una intususcepción ileocólica (figura 5). Tras la reducción manual, se visualizó una masa de $6 \mathrm{~cm}$ de diámetro, aproximadamente, en la válvula ileocecal. Se optó por una hemicolectomía oncológica derecha y anastomosis ileocólica. El periodo posoperatorio transcurrió sin complicaciones y el paciente fue dado de alta a los siete días de la intervención. El examen histopatológico de la pieza quirúrgica reveló una masa de $4 \times 6 \mathrm{~cm}$ correspondiente a un tumor del estroma gastrointestinal (GIST) (figura 6).

\section{Discusión}

La intususcepción en adultos es poco frecuente. La sospecha rara vez se confirma solamente por los hallazgos clínicos; sin embargo, se cree que cualquier lesión de la pared intestinal o cualquier irritante dentro de la luz que altere la actividad peristáltica normal, pueden iniciar una intususcepción ${ }^{9}$. Las ubicaciones más comunes son las uniones entre los segmentos que se mueven libremente y los segmentos fijos al retroperitoneo o adheridos a la cavidad peritoneal ${ }^{\text {Io }}$.

La literatura informa que el $63 \%$ de los casos de intususcepción en adultos se debe a un tumor, $50 \%$ de los cuales son malignos. Un tumor maligno es la causa en el $48 \%$ de los pacientes con intususcepción colocólica y en el I7 \% de aquellos con intususcepción entérica ${ }^{\text {Io }}$. Honjo, et al., en un estudio retrospectivo de cohorte de 16 años, documentaron que el $77,3 \%$ de los casos de intususcepción estaba relacionado con un tumor, el 73,5\% de los cuales eran malignos. Un tumor maligno es la causa en $90 \%$ de los pacientes con intususcepción colocólica y en el $25 \%$ de aquellos con intususcepción entérica ".

La presentación clínica de la intususcepción suele ser crónica e inespecífica en los adultos y la
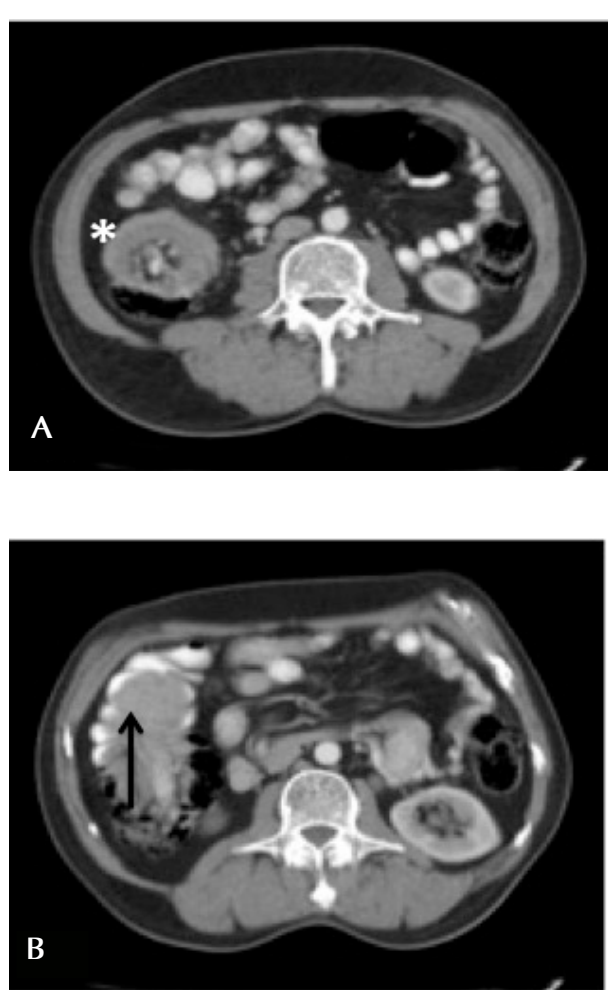

Figura 4. TC abdominal, corte axial. A. Intususcepción ileocólica con signo de la diana (asterisco). B. Origen de la intususcepción (flecha) en la válvula ileocecal y extensión de la lesión.

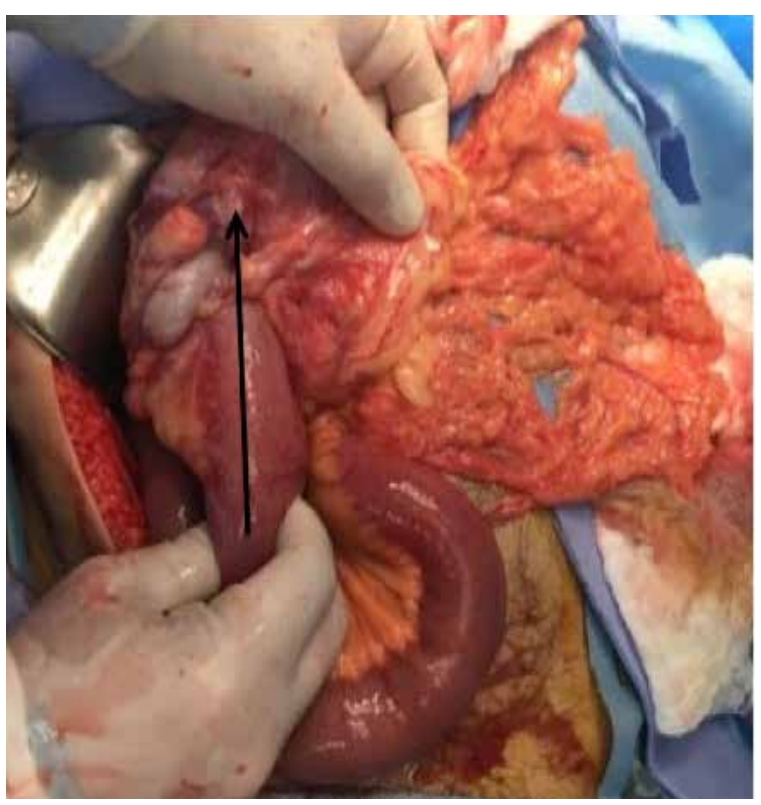

Figura 5. Pieza quirúrgica: intususcepción ileocólica (flecha) e intestino dilatado proximalmente 

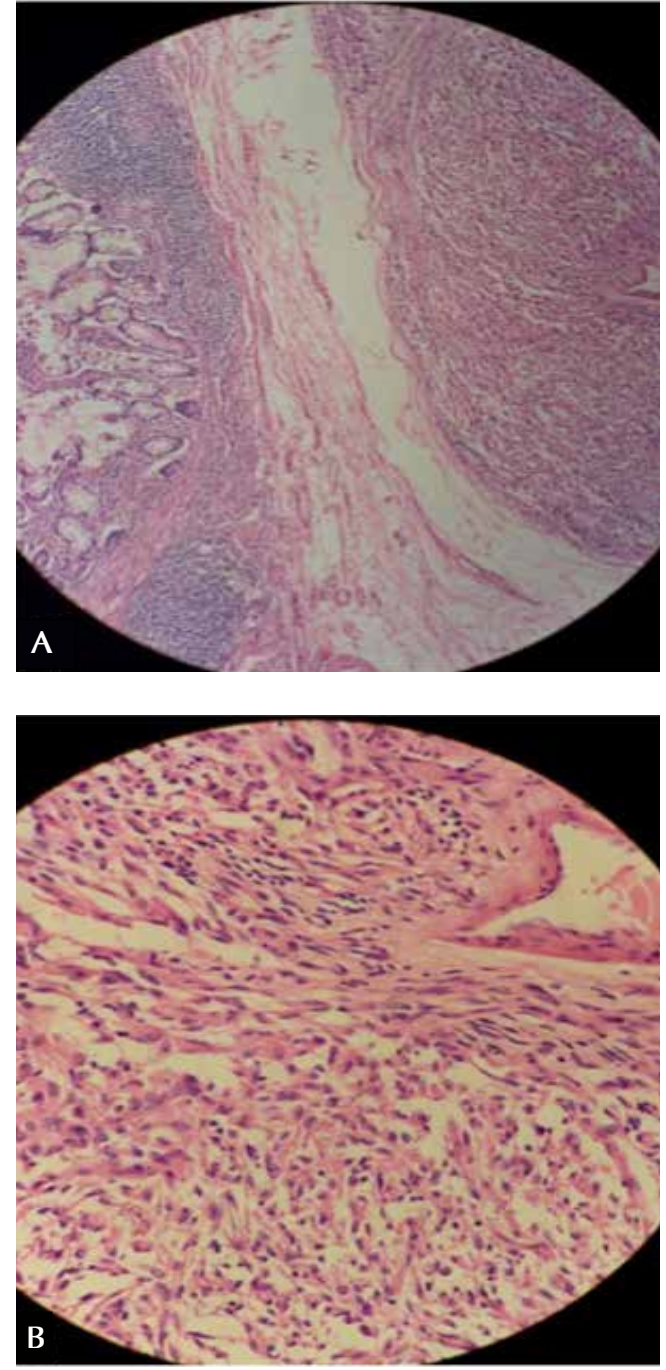

Figura 6. A. Tumor del estroma gastrointestinal (GIST). Hematoxilina y eosina, 200X B. Células tumorales en forma de huso. Hematoxilina y eosina, 40X.

mayoría presenta síntomas que sugieren obstrucción intestinal. El dolor abdominal es el síntoma más común (93,3\%), seguido del vómito y las náuseas. Es posible palpar una masa abdominal en 24 a $42 \%$ de los pacientes ${ }^{1,9}$.

En los presentes casos, el dolor abdominal fue el motivo de consulta en el servicio de urgencias y, en ninguno de los dos, se presentó la tríada clásica de la intususcepción intestinal, que consiste en dolor abdominal de tipo cólico, masa palpa- ble en forma de salchicha y heces con sangre ${ }^{12}$. Debido a que los síntomas son inespecíficos, el diagnóstico preoperatorio correcto es difícil para el cirujano, aunque, Reijnen, et al., informaron una tasa de diagnóstico preoperatorio del $50 \%^{13}$ $y$, Eisen, et al., una del 40,7\% ${ }^{14}$.

Aunque las modernas técnicas de imágenes pueden ayudar a identificar la condición, no se ha establecido un algoritmo perfecto de diagnóstico para esta enfermedad, lo que hace que el diagnóstico preoperatorio sea presuntivo. La ubicación más frecuente de las intususcepciones no está bien establecida. Wang, et al., reportaron que el 4I,7 \% son entéricas, el $50 \%$ ileocólicas y el $8,3 \%$ colocólicas ${ }^{15}$; en otro estudio, Ghaderi, et al., reportaron que la mayoría de las intususcepciones son del intestino delgado: $26,7 \%$ yeyunoyeyunal, 6,7 \% yeyunoileal, $40 \%$ ileoileal, $20 \%$ ileocólica y $6,7 \%$ colocólica ${ }^{16}$.

Entre las lesiones benignas que causan invaginación en los adultos, están los pólipos inflamatorios, los lipomas, los leiomiomas, los divertículos de Meckel y las adherencias posoperatorias ${ }^{17} \mathrm{y}$, entre las malignas, se incluyen tumores primarios como carcinoides, adenocarcinomas, pólipos malignos, tumores del estroma gastrointestinal (GIST), leiomiosarcomas, linfomas y tumores metastásicos, con mayor frecuencia los melanomas ${ }^{18-20}$.

El adenocarcinoma es la lesión cólica pura más prevalente ${ }^{15}$, lo que hace necesario que las resecciones quirúrgicas sean oncológicas, como en uno de estos casos. Sin embargo, la intususcepción es una forma de presentación muy rara de los tumores GIST, los que representan menos del $3 \%$ de todas las neoplasias malignas gastrointestinales y solo el $20 \%$ de las neoplasias entéricas, y tienden a tener un crecimiento por fuera de la luz intestinal, lo que hace que rara vez se presenten como en nuestro segundo caso ${ }^{2 \mathrm{II}}$.

\section{Estudio diagnóstico de elección}

La utilización de la tomografía computadorizada (TC) en el diagnóstico de la intususcepción, ha crecido con el paso del tiempo debido a su amplia disponibilidad. En la población pediá- 
trica, el ultrasonido es la principal herramienta diagnóstica, prueba que no se emplea en adultos por el enmascaramiento que producen las asas de intestino dilatadas por gas, ya que la principal presentación clínica es en forma de obstrucción intestinal. Por todo esto, la precisión diagnóstica del ultrasonido en los adultos es insatisfactoria ${ }^{22}$.

La TC es la modalidad de imagen de elección para el diagnóstico de intususcepción en los adultos. En esta técnica, se presenta como una masa en forma de salchicha en el corte longitudinal y como una masa en diana en el corte transversal, por el efecto de la pared intestinal y el mesenterio dentro de la luz, tal como se presenta en el segundo caso ${ }^{23}$. Su rango de precisión diagnóstica es del $58 \%$ al IOO $\%{ }^{24,25}$. Autores como Azar y Berger informan que la TC abdominal diagnostica con precisión la intususcepción intestinal en $78 \%$ de los pacientes ${ }^{\mathrm{I}}$.

La TC también proporciona otra información crítica, como la longitud y el diámetro de la intususcepción, una vista tridimensional del intestino y las vísceras circundantes, el posible punto de derivación, el tipo y la ubicación de la intususcepción intestinal, importantes puntos en el planeamiento quirúrgico ${ }^{26,27}$. Además, permite evaluar las posibles complicaciones asociadas, como obstrucción intestinal, isquemia y perforación 7 , aporta información valiosa, como la presencia de metástasis o linfadenopatías, y puede apuntar a una enfermedad subyacente ${ }^{22}$.

\section{Tratamiento de elección}

No existe un enfoque universal para el tratamiento de la intususcepción en los adultos, pero, a diferencia de los pacientes pediátricos, no se recomienda la reducción hidrostática como método de tratamiento, debido a la gran probabilidad de malignidad, por lo cual la recomendación es la resección primaria, siempre que sea posible ${ }^{28}$. Las lesiones entéricas benignas que no se acompañan de adherencias, requieren también resección para prevenir la intususcepción intestinal recurrente ${ }^{29}$.

Antes del advenimiento de las actuales modalidades de diagnóstico, la laparotomía inmediata y la resección intestinal sin reducción eran el estándar de manejo defendido por la mayoría de los cirujanos. Con el paso de los años, la controversia persiste pues algunos autores abogan por reducir la intususcepción antes de la resección para limitar su alcance, especialmente, cuando se trata de intestino delgado. No obstante, no existe 'evidencia' clara sobre este tema, ya que la reducción de la intususcepción expone a un riesgo de diseminación de células cancerígenas en la cavidad peritoneal. Por este motivo, algunos autores siguen recomendando la resección primaria sin reducción ${ }^{30-32}$.

Que el tipo de abordaje quirúrgico sea laparoscópico o abierto, depende de varios factores: la ubicación de la intususcepción, el grado de intususcepción u obstrucción intestinal asociado, la viabilidad del intestino en el momento del acto quirúrgico, la condición clínica del paciente y, en particular, la experiencia del cirujano en procedimientos laparoscópicos ${ }^{30,33}$. Actualmente, se carece de evidencia científica que recomiende una u otra técnica, ya que la mayoría de los informes se basan en reporte de casos, series y evidencia anecdótica. Si no hay obstrucción intestinal, la laparoscopia podría ser el método de elección para el abordaje quirúrgico. El pronóstico de la intususcepción está relacionado con la enfermedad de base ${ }^{31}$.

\section{Conclusión}

La intususcepción en adultos es inusual y desafiante, y requiere de habilidad diagnóstica preoperatoria. Los cirujanos deben estar familiarizados con las diversas opciones de tratamiento y los diferentes posibles abordajes, y siempre que sea posible, se debe hacer una resección primaria. La verdadera causa de la intususcepción se diagnostica con precisión mediante el estudio histopatológico posterior.

Conflicto de intereses. Los autores no tienen potencial conflicto de intereses con respecto a la publicación de este documento.

Fuentes de financiación. Autofinanciado 


\section{Referencias}

I. Azar T, Berger DL. Adult intussusception. Ann Surg. I997;226:I34-8. doi: I0.4253/wjge.v9.i5.220

2. Shenoy S. Adult intussusception: A case series and review. World J Gastrointest Endosc. 20I7;9:220-227. doi: I0.4253/wjge.v9.i5.220

3. Marinis A, Yiallourou A, Samanides L, Dafnios N, Anastasopoulos G, Vassiliou I, et al. Intussusception of the bowel in adults: A review. World J Gastroenterol. 2009;15:407-II.

4. Sarr MG, Nagorney DM, McIlrath DC. Postoperative intussusception in the adult: A previously unrecognized entity? Arch Surg. 198I;II6:I44-8.

5. Kuppermann N, O’Dea T, Pinckney L, Hoecker C. Predictors of intussusception in young children. Arch Pediatr Adolesc Med. 2000;154:250-5.

6. Kim YH, Blake MA, Harisinghani MG, Archer-Arroyo K, Hahn PF, Pitman MB, et al. Adult intestinal intussusception: CT appearances and identification of a causative lead point. Radiographics. 2006; 26:733-44. doi: I0.II48/rg.263055I00

7. Onkendi EO, Grotz TE, Murray JA, Donohue JH. Adult intussusception in the last 25 years of modern imaging: Is surgery still indicated? J Gastrointest Surg. 20II;I5:I699-705. doi: I0.I007/sII605-OII-I609-4

8. Rea JD, Lockhart ME, Yarbrough DE, Leeth RR, Bledsoe SE, Clements RH. Approach to management of intussusception in adults: A new paradigm in the computed tomography era. Am Surg. 2007;73:I098-IIO5.

9. Begos DG, Sandor A, Modlin I. The diagnosis and management of adult intussusception. Am J Surg. I997;173:88-94. doi: I0.IOI6/Sooo2-96IO(96)00419-9

Io. Sachs M, Encke A. Entero-enteral invagination of the small intestine in adults. A rare cause of "uncertain abdomen". Langenbecks Arch Chir.1993;378:288-9I.

II. Honjo H, Mike M, Kusanagi H, Kano N. Adult intussusception: A retrospective review. World J Surg. 2015;39:134-8. doi: I0.1007/soo268-0I4-2759-9

I2. Felix EL, Cohen MH, Bernstein AD, Schwartz JH. Adult intussusception: Case report of recurrent intussusception and review of the literature. Am J Surg. 1976;131:758$76 \mathrm{I}$.

I3. Reijnen HA, Joosten HJ, DeBoer HH. Diagnosis and treatment of adult intussusception. Am J Surg. I989;I58:25-8.

I4. Eisen LK, Cunningham JD, Aufses AH Jr. Intussusception in adults: Institutional review. J Am Coll Surg. I999;188:390-5.

I5. Wang LT, Wu CC, Yu JC, Hsiao CW, Hsu CC, Jao SW. Clinical entity and treatment strategies for adult intussusceptions: 20 years' experience. Dis Colon Rectum. 2007;50:194I-9. doi: I0.1007/sI0350-007-9048-8

I6. Ghaderi H, Jafarian A, Aminian A, Mirjafari Daryasari SA. Clinical presentations, diagnosis and treat- ment of adult intussusception, a 20 years survey. Int J Surg. 20I0;8:3I8-20. doi: I0.IOI6/j.ijsu.20I0.02.013

I7. Ludvigsson JF, Nordenskjöld A, Murray JA, Olén O. A large nationwide population-based case-control study of the association between intussusception and later celiac disease. BMC Gastroenterol. 2013;13:89. doi: IO.II86/I47I-230X-I3-89

I8. Erkan N, Haciyanli M, Yildirim M, Sayhan H, Vardar E, Polat AF. Intussusception in adults: An unusual and challenging condition for surgeons. Int J Colorectal Dis. 2005;20:452-456. doi: I0.I007/s00384-004-0713-2

19. Shenoy S, Cassim R. Metastatic melanoma to the gastrointestinal tract: Role of surgery as palliative treatment. West Virginia Med J. 2013;109:30-3.

20. Shenoy S. Small bowel metastases: Tumor markers for diagnosis and role of surgical palliation. J Gastrointest Cancer. 20I6;47:210-13.

2I. Gupta A, Gupta S, Tandon A, Kotru M, Kumar S. Gastrointestinal stromal tumor causing ileo-ileal intussusception in an adult patient: A rare presentation with review of literature. Pan Afr Med J. 20II;8:29-34. doi: IO.II604/pamj.2OII.8.29.424

22. Gupta RK, Agrawal CS, Yadav R, Bajracharya A, Sah PL. Intussusception in adults: Institutional review. Int J Surg. 20II;9:9I-5. doi: I0.IOI6/j.ijsu.20I0.10.003

23. Park SB, Ha HK, Kim AY, Lee SS, Kim HJ, Park BJ, et al. The diagnostic role of abdominal CT imaging findings in adults intussusception: Focused on the vascular compromise. Eur J Radiol. 2007;62:406-I5. doi: I0.IOI6/j. ejrad.2007.0I.003

24. Barussaud M, Regenet N, Briennon X, de Kerviler B, Pessaux P, Kohneh-Sharhi N, et al. Clinical spectrum and surgical approach of adult intussusceptions: A multicentric study. Int J Colorectal Dis. 2006;21:834-9. doi: $10.1007 /$ so0384-005-0789-3

25. Gayer G, Zissin R, Apter S, Papa M, Hertz M. Pictorial review: Adult intussusception-a CT diagnosis. $\mathrm{Br} \mathrm{J}$ Radiol. 2002;75:185-90.

26. Gore RM, Silvers RI, Thakrar KH, Wenzke DR, Mehta UK, Newmark GM, et al. Bowel obstruction. Radiol Clin North Am. 2015;53:1225-40. doi: I0.IOI6/ j.rcl.2015.06.008

27. Gayer G, Apter S, Hofmann C, Nass S, Amitai M, Zissin $\mathrm{R}$, et al. Intussusception in adults: CT diagnosis. Clin Radiol. 1998;53:53-7.

28. Barbiera F, Cusma S, Di Giacomo D, Finazzo M, Lo Casto A, Pardo S. Adult intestinal intussusception: Comparison between CT features and surgical findings. Radiol Med. 200I;IO2:37- 42.

29. Takeuchi K, Tsuzuki Y, Ando T, Sekihara M, Hara $\mathrm{T}$, Kori $\mathrm{T}$, et al. The diagnosis and treatment of adult intussusception. J Clin Gastroenterol. 2003;36:I8-2I.

30. Ishibashi Y, Yamamoto S, Yamada Y, Fujita S, Akasu T, Moriya Y. Laparoscopic resection for malignant lymphoma of the ileum causing ileocecal intussusception. Surg Laparosc Endosc Percutan Tech. 2007;17:444-6. 
3I. Jelenc F, Brencic E. Laparoscopically assisted resection of an ascending colon lipoma causing intermittent intussusception. J Laparoendosc Adv Surg Tech. 2005;I5:I73-5. doi: I0.I089/lap.2005.I5.I73

32. Maghrebi H, Makni A, Rhaiem R, Atri S, Ayadi M, Jrad $\mathrm{M}$, et al. Adult intussusceptions: Clinical presentation, diagnosis and therapeutic management. Int J Surg Case Rep. 20I7;33:I63-6. doi: I0.IOI6/j.ijscr.20I7.02.009

33. Arslan E, Çağlayan K, Sipahi M, Banli O, Gündoğdu F, Şahin S. Intussusception of the bow el in adults: Two different cases. Turk J Surg. 2015;33:2179. doi: I0.5I52/UCD.20I5.300I 